\title{
Chaotic synchronization of coupled electron-wave systems with backward waves
}

\author{
Alexander E. Hramov * Alexey A. Koronovskii $\mathbb{A}$ Pavel V. Popov, and Irene S. Rempen \\ Faculty of Nonlinear Processes, Saratov State University, Astrakhanskaya, 83, Saratov, 410012, Russia
}

(Dated: November 21, 2018)

\begin{abstract}
The chaotic synchronization of two electron-wave media with interacting backward waves and cubic phase nonlinearity is investigated in the paper. To detect the chaotic synchronization regime we use a new approach, the so-called time scale synchronization [Chaos, 14 (3) 603-610 (2004)]. This approach is based on the consideration of the infinite set of chaotic signals' phases introduced by means of continuous wavelet transform. The complex space-time dynamics of the active media and mechanisms of the time scale synchronization appearance are considered.
\end{abstract}

PACS numbers: 05.45.Xt, 05.45.Tp

Keywords: coupled oscillators, spatially extended system, space-time chaos, chaotic synchronization, phase, wavelet transform, microwave tubes

Synchronization of chaotic oscillators is one of the fundamental phenomena of nonlinear dynamics. In this paper we consider chaotic synchronization of two electron-wave media with interacting backward waves and cubic phase nonlinearity. An efficient approach to detecting of synchronous dynamics of distributed systems is suggested based on the time scale synchronization concept [Chaos, 14 (3) 603-610 (2004)]. The complex space-time dynamics of the active media and mechanisms of the appearance of time scale synchronization are discussed.

\section{INTRODUCTION}

In last decade the investigation of chaotic synchronization has become a task of great interest [1, 2, 3, 4]. It can be observed in a lot of chaotic oscillators of different nature, including physical and biological systems 4, 5, 6, 7, 8]. Chaotic synchronization can be used for secret signal transmission [9, 10, 11, 12. It also seems very important to study the appearance of chaotic synchronization in distributed systems demonstrating spacetime chaos [13, 14, 15, 16, 17, 18, 19]. Four types of chaotic synchronization have been carried out for chaotic systems with lumped parameters, namely phase synchronization (PS), complete synchronization (CS), lag synchronization (LS) and generalized synchronization (GS) 22, 3, 4, 20]. On the ranges of mentioned regimes the intermittence behaviour 21] takes place. To describe the phase synchronization the instantaneous phase $\phi(t)$ of a chaotic continuous time series is usually introduced (see [1, 2, 4, 22]). The PS means the entrainment of phases of chaotic signals, whereas their amplitudes remain chaotic and uncorrelated. Simultaneously, if Fourier spectrum of a signal $x(t)$ is complex (chaotic time series are character-

*Electronic address: aeh@cas.ssu.runnet.ru

${ }^{\dagger}$ Electronic address: alkor@cas.ssu.runnet.ru ized by Fourier spectrum without the main frequency), it is not always possible to describe the system dynamics with the help of one instantaneous phase $\phi(t)$ (see 23, 24] for detail). In this case other methods can be applied to analyze the non-phase coherent complex systems dynamics (e.g., via curvature or via recurrence, see [25, 26] for detail).

In our works [24, 27] the new approach to the analysis of the chaotic synchronization is described, based on examination of continuous set of phases $\phi_{s}(t)$ of chaotic time series introduced with the help of the continuous wavelet transform [28, 29, 30].

Let us consider continuous wavelet transform of time series $x(t)$

$$
W\left(s, t_{0}\right)=\int_{-\infty}^{+\infty} x(t) \psi_{s, t_{0}}^{*}(t) d t,
$$

where $\psi_{s, t_{0}}(t)$ is the wavelet-function related to the mother-wavelet $\psi_{0}(t)$ as

$$
\psi_{s, t_{0}}(t)=\frac{1}{\sqrt{s}} \psi\left(\frac{t-t_{0}}{s}\right) .
$$

In equation (11) $x(t)$ is the time series generated by a dynamical system. It may also contain the noise component. The time scale $s$ corresponds to the width of the wavelet function $\psi_{s, t_{0}}(t)$, and $t_{0}$ is the shift of wavelet along the time axis, symbol " $*$ " in (1) denotes complex conjugation. It should be noted that the time scale $s$ is usually used instead of the frequency $f$ of Fourier transform and can be considered as the quantity inversed to it.

The Morlet-wavelet 31

$$
\psi_{0}(\eta)=\frac{1}{\sqrt[4]{\pi}} \exp \left(j \Omega_{0} \eta\right) \exp \left(\frac{-\eta^{2}}{2}\right)
$$

has been used as a mother-wavelet function. The choice of parameter value $\Omega_{0}=2 \pi$ provides the relation $s=1 / f$ between the time scale $s$ of wavelet transform and frequency $f$ of Fourier transform. 
The wavelet surface

$$
W\left(s, t_{0}\right)=\left|W\left(s, t_{0}\right)\right| e^{j \phi_{s}\left(t_{0}\right)}
$$

describes the system's dynamics on every time scale $s$ at moment $t_{0}$. The value of $\left|W\left(s, t_{0}\right)\right|$ indicates the presence and intensity of the time scale $s$ mode in the time series $x(t)$ at the moment of time $t_{0}$. It is also possible to consider the quantity

$$
\langle E(s)\rangle=\int\left|W\left(s, t_{0}\right)\right|^{2} d t_{0},
$$

which is the distribution of integral energy by time scales.

At the same time, the phase $\phi_{s}(t)=\arg W(s, t)$ is naturally introduced for every time scale $s$. In other words, $\phi_{s}(t)$ is a continuous function of time $t$ and time scale $s$. It means that it is possible to describe the behavior of each time scale $s$ by means of its own phase $\phi_{s}(t)$. Let us consider the dynamics of two coupled oscillators. If in the time series $\mathbf{x}_{1,2}(t)$ generated by these systems there is a range of time scales $s_{1} \leq s \leq s_{2}$ for which the phase locking condition

$$
\left|\phi_{s 1}(t)-\phi_{s 2}(t)\right|<\mathrm{const}
$$

is satisfied and the part of the wavelet spectrum energy in this range is not equal to zero

$$
E_{s n h r}=\int_{s_{1}}^{s_{2}}\langle E(s)\rangle d s>0,
$$

then we assert that time scale synchronization (TSS) between oscillators takes place 24]. The condition (6) can be generalized to the case of $m: n$ synchronization. In this case one has to use the condition

$$
\left|m \phi_{s n 1}(t)-n \phi_{s m 2}(t)\right|<\mathrm{const}
$$

instead of (6). In this case the time scales $s_{m 1}$ in the first system and $s_{n 2}$ in the second system would satisfy the relation $s_{m 2} / s_{n 1}=m / n$.

In Reference 24] the new approach was applied to the study of chaotic synchronization of coupled Rössler systems and two Chua's systems. It was shown, that different types of synchronous oscillations (PS, LS, ILS, CS and GS) may be considered as particular cases of TSS. It seems rather interesting to apply the concept of time scale synchronization to the analysis of chaotic synchronization of the distributed systems demonstrating spacetime chaos.

In this paper we present the results of investigation of chaotic synchronization in the coupled electron-wave systems with backward electromagnetic wave and cubic nonlinearity, derived with the help of the method of time scale synchronization. The explored system is a simple model of microwave oscillator with backward wave (BWO) finding wide practical application (see, for example, 32, 33, 34, 35]). For similar systems the complex chaotic regimes of space-time oscillations are typical [36, 37, 38, 39, 40, 41].
The structure of the paper is as follows. Section [II contains the formalism describing nonlinear interaction of electron beam and backward electromagnetic wave. Section III describes the synchronization of two unidirectional coupled electron-wave systems. We demonstrate the efficiency of TSS method for the analysis of synchronization of spatially extended systems and discuss the characteristics of chaotic synchronization of such systems. The quantitative measure of synchronization of spatially extended systems is described in section IV In section $\mathbf{D}$ we consider complex space-time dynamics of the unidirectional coupled electron-wave systems with the help of the concept of time-scale synchronization. The final conclusion is presented in section VI]

\section{GENERAL FORMALISM}

Let us consider a simple model of two bound electronwave media with backward wave and cubic phase nonlinearity.

One of the types of the active media of electron-wave nature is based on resonance interaction of electron wave and backward electromagnetic wave in the slow-wave structure [32, 33, 34, 42]. The electron wave is really a perturbation of density and velocity of charged particles of electron beam propagating with the velocity $v_{0}$ along a slow-wave structure.

In the linear approximation such system conforms to a model of interaction of two opposing linear waves with dispersion relation

$$
\omega_{1}=k_{1}, \quad \omega_{2}=-k_{2} .
$$

In this case the linear model can be formulated as a selfconsistent system of two equations

$$
\begin{aligned}
& \frac{\partial F}{\partial \tau}-\frac{\partial F}{\partial \xi}=-A I, \\
& \frac{\partial I}{\partial \tau}+\frac{\partial I}{\partial \xi}=-A F
\end{aligned}
$$

where an absolute instability is observed. Here $F=$ $|F| \exp \left(j \varphi_{F}\right)$ and $I=|I| \exp \left(j \varphi_{I}\right)$ are the normalized slowly variable amplitudes of interacting electromagnetic and electron waves, respectively, $\tau$ is the normalized time, $\xi$ is the normalized coordinate and $A$ is the normalized interaction parameter, which is proportional to the electron beam current and the length of the active medium.

Solution of the linear equations (10) and (111) predicts unlimited exponential growth of amplitude of each of the waves. In this case it is necessary to take into account the nonlinear effects. One of the fundamental nonlinear effects is the non-isochronism of electrons-oscillators, expressed in dependence of the frequency of the electronoscillator on its energy. Non-isochronism of electronsoscillators in the system of interacting waves is displayed 
in the shift of nonlinear phase $\varphi_{I}$ of the electron wave. Replacing the old variables in the equations (10) and (11) to new ones: $\tau^{\prime}=(\tau-\xi) / 2$ and $\xi^{\prime}=\xi$ we can rewrite (11) as

$$
\frac{\partial I}{\partial \xi}+j|I| \frac{\partial \varphi_{I}}{\partial \xi}=-A|F| \exp \left(j\left(\varphi_{F}-\varphi_{I}\right)\right),
$$

where primes above new variables are omitted.

From the point of view of the non-isochronism effect the expression for $\partial \varphi_{I} / \partial \xi$ can be obtained from the following reasons. The equation for the complete phase $\varphi_{I}$ of electron wave must contain the item $\left(\omega_{e} / v_{e}\right) \xi$, defining the shift of the frequency of radiation due to the Doppler effect (here $\omega_{e}$ is the frequency of the electron wave and $v_{e}$ is the longitudinal velocity of its propagation). So, when we take into account the dependence of $\omega_{e}$ upon the energy of the particles $\mathcal{W}$, the item proportional to energy $\mathcal{W}$ should be added at first approximation into the expression for a slow phase:

$$
\omega_{e}=\omega_{e 0}+\left(\frac{\partial \omega_{e}}{\partial \mathcal{W}}\right)_{0} \mathcal{W}+\ldots
$$

Supposing, that non-perturbed frequency $\omega_{e 0}$ is taken into account in the "fast" phase, and the energy $\mathcal{W}$ of electrons (in case when their trajectories are identical) is defined by the energy of the wave by the expression $\mathcal{W}=\alpha|I|^{2}$, where $\alpha$ is the coefficient of proportionality, we shall discover, that

$$
\frac{\partial \varphi_{I}}{\partial \xi}=\frac{\partial \varphi_{I}^{\prime}}{\partial \xi}+\left(\frac{\partial \omega_{e}}{\partial \mathcal{W}}\right)_{0} \mathcal{W} \frac{\alpha|I|^{2}}{v_{e}}=\frac{\partial \varphi_{I}^{\prime}}{\partial \xi}+\mu|I|^{2},
$$

where the derivative $\partial \varphi_{I}^{\prime} / \partial \xi$ describes the phase shift of the wave only due to the interaction between electrons and electromagnetic wave $F ; \mu=\left(\alpha / v_{e}\right)\left(\partial \omega_{e} / \partial \mathcal{W}\right)_{0} \mathcal{W}$ is the parameter characterizing the change of the electron velocity agreeing with the change of the electron energy during the interaction. We shall now define the amplitude of an electron wave as $I=|I| \exp \left(j \varphi_{I}^{\prime}\right)$ and deal with the new variable $F^{\prime}=F / \sqrt{\mu}, I^{\prime}=I / \sqrt{\mu}$, so the equation (12) will be written as follows

$$
\frac{\partial I}{\partial \xi}+j|I|^{2} I=-A F,
$$

where the primes above new variable are omitted.

Thus, the equation (10) for the backward electromagnetic wave will not change.

The equations (10) and (15) describe the nonlinear oscillations in the microwave spatially extended system of interacting backward waves with cubic phase nonlinearity. Such electron-wave system, as wtitten above, is a simple model of microwave oscillator with backward wave (BWO 32, 33] and gyrotron BWO [33, 35, 39]). This system is suitable for the analysis of chaotic oscillations, including chaotic synchronization, in microwave tubes (see, for example, BWO 36, 37, 38 and gyrotron backward wave generator [35, 39]).
In an autonomous regime of BWO it is necessary to supplement the equations (10) and (15) with the standard boundary conditions $F(\xi=1, \tau)=0$ and $I(\xi=$ $0, \tau)=0$ describing the lack of electromagnetic and electron waves on the boundaries of the autonomous system. In the autonomous microwave oscillator with backward wave at $\pi / 2<A<1.83$ the single-frequency oscillations with stationary space distribution of electromagnetic field $F(\xi)$ and current $I(\xi)$ are observed. At $A>1.83$ the multifrequency periodic oscillations (regime of a periodic self-modulation of an output field [37, 38, 39]) take place. At $A>2.05$ the time series of the output field $F(\tau, \xi=0)$ look like pulses with fine oscillations between them, as a result of excitation of the complex irregular space distribution $F(\xi)$ and $I(\xi)$. The reason of the latter is the fast shift of the nonlinear phase of the electron wave along the space coordinate of the distributed system. At last, at $A>4.5$ chaotic wide-band oscillations are observed.

In present paper we consider the system of two unidirectionally coupled, spatially extended electron-wave systems in the chaotic regime. The system is described by the following equations

$$
\begin{gathered}
\frac{\partial F_{1,2}}{\partial \tau}-\frac{\partial F_{1,2}}{\partial \xi}=-A_{1,2} I_{1,2}, \\
\frac{\partial I_{1,2}}{\partial \xi}+j\left|I_{1,2}\right|^{2} I_{1,2}=-A_{1,2} F_{1,2},
\end{gathered}
$$

where index " 1 " corresponds to the drive system and index "2" - to the response one.

Unidirectional connection between microwave oscillators is brought into the system as a non-stationary boundary condition for the slowly varying amplitude of the electromagnetic field $F_{2}$ of the response system thus the boundary condition for the first conducting fissile medium remains constant

$$
\begin{gathered}
I_{1}(\xi=0, \tau)=0, \quad F_{1}(\xi=1, \tau)=0, \\
I_{2}(\xi=0, \tau)=0, \quad F_{2}(\xi=1, \tau)=\rho F_{1}(\xi=0, \tau) .
\end{gathered}
$$

Hence $\rho=R \exp [j \theta]$ is the complex coupling coefficient ( $R$ is the coupling strength and $\theta$ is the phase of coupling coefficient).

For drive and response systems the values of control parameters are taken as $A_{1}=4.2$ and $A_{2}=4.9$, that corresponds to the regimes of chaotic oscillations in the active electron-wave systems with backward waves. The highest Lyapunov exponents are $\lambda_{A 1}=0.224 \pm 0.010$ and $\lambda_{A 2}=0.617 \pm 0.006$ for the first and second control parameter values, respectively. The value of the phase of coupling coefficient $\theta$ does not influence the processes in the coupled systems and is fixed $\theta=\pi$. In present paper the establishment of the chaotic synchronization regime in two spatially extended electron-wave systems (BWO) is analyzed at the variation of coupling strength $R$. 

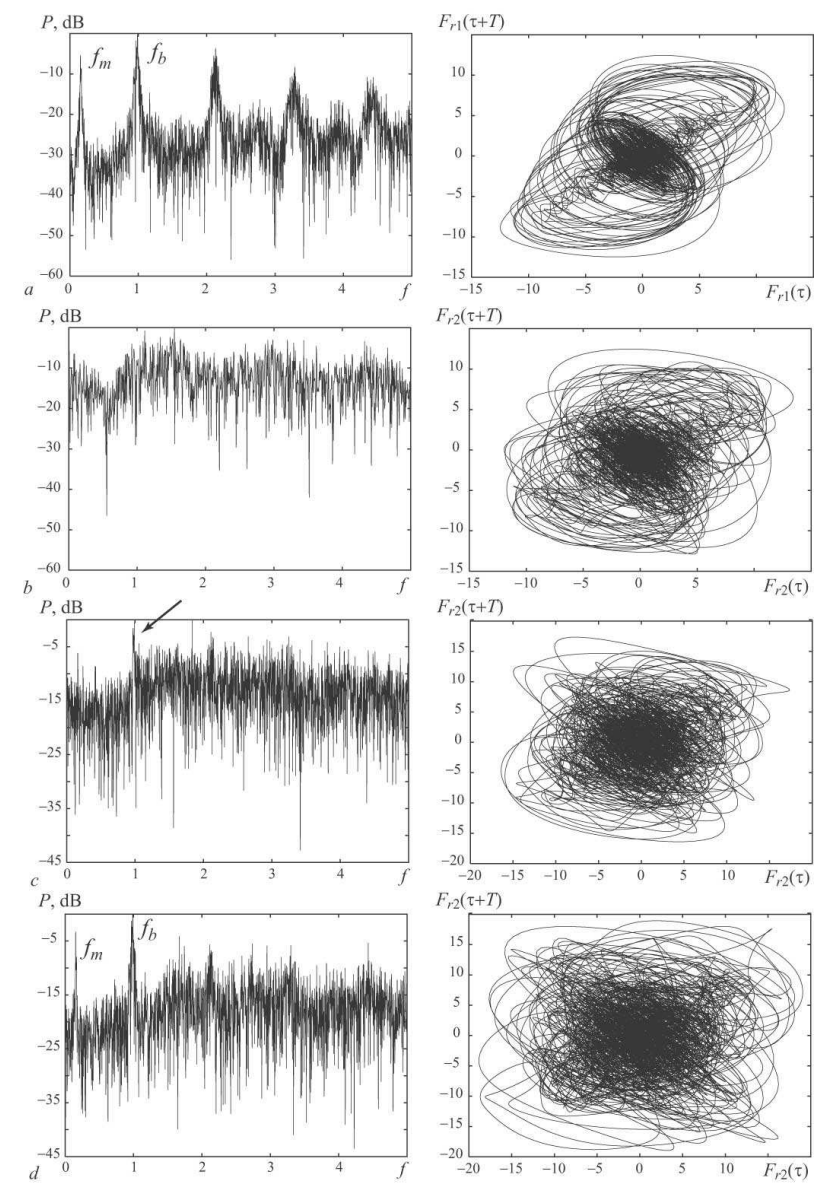

FIG. 1: Power spectra and phase pictures of output electromagnetic field oscillations. (a) Drive oscillator with the value of dimensionless parameter $A=4.2$. (b) Response oscillator without external signal $(A=4.9$ and $R=0)$. (c,d) Response oscillator with the external signal. The value of coupling parameter $R$ is (c) $R=0.2$ and (d) $R=0.5$

\section{CHAOTIC SYNCHRONIZATION OF SPATIALLY EXTENDED ELECTRON-WAVE SYSTEMS}

Let us consider the behavior of the coupled systems with the increase of amplitude of coupling coefficient $R$ and the fixed values of control parameters (the values mentioned above are taken). We shall investigate the oscillations $F_{r 1,2}(\tau)=\operatorname{Re}\left[F_{1,2}(\xi=0, \tau)\right]$ at outputs $\xi=$ 0 of both coupled systems.

Figures [1, and show the power spectra and phase pictures (reconstructed by delay-coordinate embedding method [43, 44]) of oscillations at the outputs of drive and response systems in autonomous regimes (i.e. at $R=0)$.

The output field dynamics $F_{r 1}$ of the drive oscillator (which acts upon the input of the interaction space $\xi=1$ of response oscillator) is chaotic (see Fig. 1n). In the power spectrum the high noise background at the level $-30 \mathrm{~dB}$ is observed. There are two main spectral com-

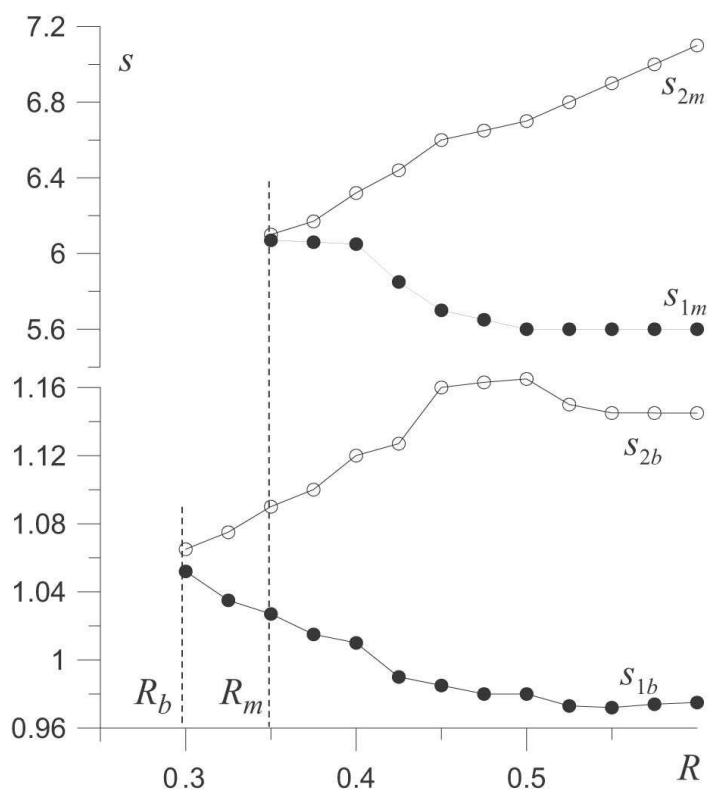

FIG. 2: The dependence of the synchronized time scale range $\left[s_{1} ; s_{2}\right]$ on the coupling strength $R$ for base time scales $s_{b}$ (bottom) and $s_{m}$ (top)

ponents with frequencies $f_{b}$ and $f_{m}$ in this spectrum. High frequency $f_{b}$ is close to frequency $\hat{\omega} / 2 \pi$ of synchronism of the linear noninteracting electron and backward electromagnetic waves. At the decrease of dimensionless parameter of the electron beam $A_{1}$ the regime of periodic oscillations with the frequency close to $f_{b}$ is established. The second frequency which is marked as $f_{m}$ and is settling down in a low-frequency part of a power spectrum, determines the low-frequency modulation of complex amplitude $\left|F_{1}(\tau)\right|$ of the electromagnetic backward wave.

With the growth of control parameter $A$ the oscillations in the electron-wave system complicate, that is obviously seen from the analysis of the power spectrum and the chaotic attractor in Fig. I ( b), of the second system at $A=4.9$ and $R=0$ (autonomous oscillations). It is shown in Fig. (1) b), that the noise background of the power spectrum rises up to a level $-(5 \div 10) \mathrm{dB}$. With such noise background it is already impossible to select the main spectral components.

Let us consider the behavior of the response system under the influence of the drive system signal, at the increase of coupling coefficient $R$. The growth of the coupling strength parameter $R$ means that the power of external signal acting on the response system, increases as $R^{2}$.

With the growth of a coupling parameter $R$ the power spectrum of generation of the response system changes that is illustrated by Fig.1(c,d). At the small $R<0.3$ [Fig.1(c)] upon the noise base of the response system spectrum there is a weakly delineated frequency (marked by an arrow), corresponding to the base frequency $f_{b}$ of the driving system spectrum [see Fig. [1(a)]. At the large values $R>0.3$ the energy of the spectral component 
corresponding to the base frequency (marked by arrow in Fig. 1(d); $R=0.5$ ) increases.

It is necessary to mark, that both systems have attractors with ill-defined phase. For these chaotic signals it is impossible to introduce the instantaneous phase $\phi(t)$ of chaotic signal correctly. It is clear that in such case the traditional methods of the phase synchronization detecting (see Ref. 11, 2, 22, 23]) fail. On the contrary, the approach based on introduction of instantaneous phase $\phi_{s}(t)$ by the wavelet transform [24, 27] can easily help to detect the time scale synchronization between chaotic oscillators.

The dependence of synchronized time scale range $\left[s_{1} ; s_{2}\right]$ on coupling parameter $R$ is shown in Fig. 2 The appearance of the synchronized time scale range corresponds to the TSS regime.

The peculiarity of dynamics of the system of two unidirectional coupled spatially extended systems is that the TSS of chaotic oscillations of each of subsystems at a large value of coupling parameter $R$ is observed for two ranges near to base time scales $s_{b}=1 / f_{b}$ and $s_{m}=1 / s_{m}$, respectively. In Fig. 2 the ranges of phase locked scales are shown for the areas near to the base time scale $s_{b}$ (lower figure), and the time scale $s_{m}$ (upper figure).

From Fig.2] it can be seen, that for coupling coefficient values $R<0.29$ the establishment of a chaotic synchronization regime is not observed. The latter means, that there are no time scales $s$ which would satisfy the conditions of TSS (6) and (7).

Figure 3 illustrates the dynamics of phase difference $\phi_{s 1}(t)-\phi_{s 2}(t)$ for different time scales for $R=0.2$. It is clear, that there is no time scale range $\left[s_{1} ; s_{2}\right]$ for which the phase difference $\phi_{s 1}(t)-\phi_{s 2}(t)\left(s \in\left[s_{1} ; s_{2}\right]\right)$ is bounded.

For the coupling parameter $R$ exceeding the critical value $R_{c} \simeq 0.29$ we observe the time scale range $\Delta s=$ $s_{2}-s_{1}$ for which the requirement of capture of phases (66) is satisfied and the energy relating on this range (7) is nonzero. The latter means, that in the coupled active media the TSS regime is established [24, 27].

The behavior of the phase difference $\phi_{s 1}(t)-\phi_{s 2}(t)$ for this case is presented in Fig. [3. (b). So, we can say, that the time scales $s=1.055$ (and close to them) of chaotic oscillations of electromagnetic field amplitudes on the output of coupled systems are synchronized with each other. At the same time the other time scales (e.g., $s=1.5)$ remain uncorrelated. For such time scales the phase locking is not observed [see Fig. 3(b)].

Let us note, that with the growth of coupling parameter the TSS appears, at first, near the base scale $s_{b}$ at $R=R_{b}$ (it is marked in Fig. 22). With the further increase of coupling coefficient, at $R=R_{m}>R_{b}$ the phase locking of time scales occurs in the area of a time scale $s_{m}$, corresponding to modulating frequency $f_{m}$ of amplitude of the output field of the response BWO.

Let us consider at first, why the range $\Delta s$ of synchronized time scales (i.e. time scales, for which the conditions of synchronization (6) and (17) are fulfilled) ap-

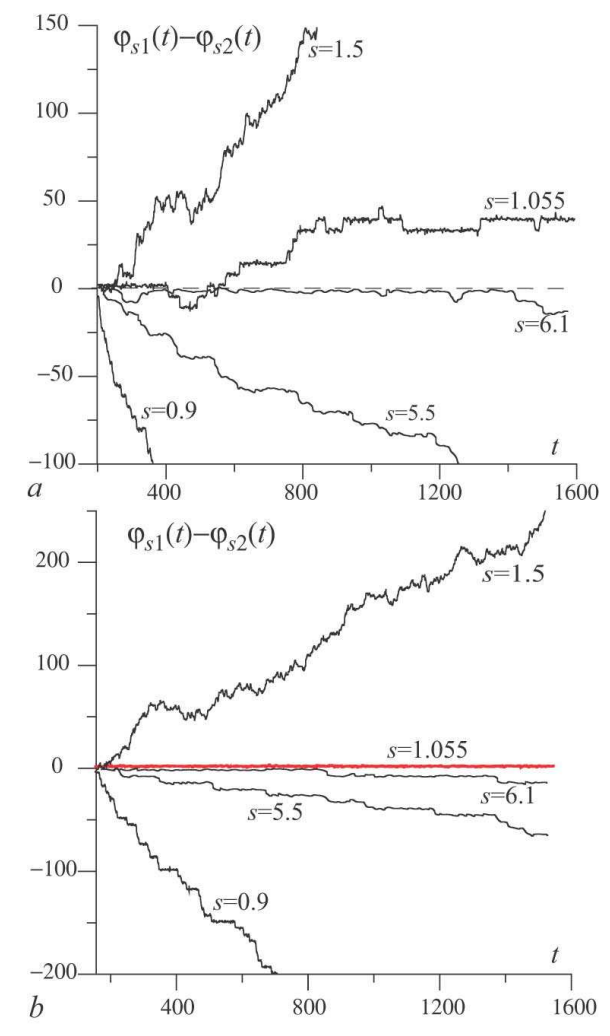

FIG. 3: The phase difference $\phi_{s 1}(t)-\phi_{s 2}(t)$ for two unidirectionally coupled spatially extended electron-wave systems. (a) $R=0.2$. There is no synchronization between systems. (b) $R=0.3$. The time scales $s=1.055$ (red line) of both systems are correlated with each other and the time scale synchronization regime can be observed

pears near the time scale $s_{b}$ corresponding to the base frequency $f_{b}=1 / s_{b}$. For this purpose we shall study wavelet power spectra $\left\langle E_{1,2}(s)\right\rangle$, defined from the equation (51), for drive and response systems. Wavelet spectra of the output field oscillations of drive (a stroke line 1) and response (a solid line 2) BWO are presented in Fig.4 for different values of coupling parameter $R$. Let us mark, that the wavelet power spectrum $\left\langle E_{1}(s)\right\rangle$ of the drive system does not vary (since the value of controlling parameter $A_{1}$ is fixed and the system is autonomous) and is presented in all the figures, for different values of $R$, as a matter of convenience comparison with the wavelet power spectra $\left\langle E_{2}\right\rangle$ of the response system.

In Fig. प(a) wavelet power spectra of autonomous oscillations are shown $(R=0)$. One can see that the oscillations in each of the spatially extended systems have essentially different wavelet power spectra. So, in wavelet spectrum $\left\langle E_{1}(s)\right\rangle$ of the drive system the base time scales $s_{b}$ (corresponding to the high frequency $f_{b}$ ) and $s_{m}$ (corresponding to modulation oscillations with the frequency $\left.f_{m}=1 / s_{m}\right)$ stand out against the background. In wavelet spectrum of an autonomous response system the time scales corresponding to high-frequency spectral component (they are marked by an arrow in Fig. प(a)) 

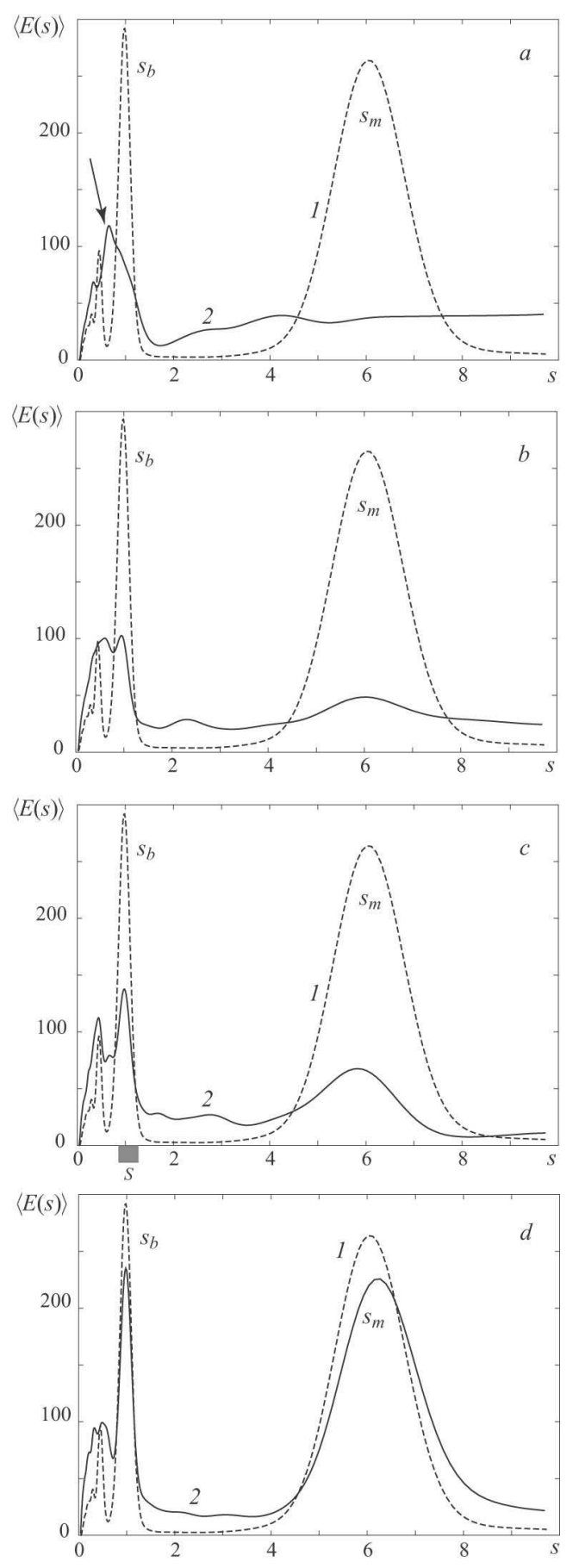

FIG. 4: The energy distribution in wavelet spectra $\langle E(s)\rangle$ for output field oscillations of drive (stroke line) and response (solid line) spatially extended systems. (a) $R=0$. There are autonomous oscillations in the systems. (b) $R=0.2$. There is no time scale synchronization between systems. (c) $R=0.3$. There is time scale synchronization near the base time scale $s_{b}$ (Synchronized scale region is marked by gray box $S$ ). (d) $R=0.5$. Time scale synchronization near both base time scale $s_{b}$ and modulation time scale $s_{m}$ is observed. also precisely stand out against background. However, the arrangement of a peak of $s_{b}$ in wavelet power spectrum $\left\langle E_{2}(s)\right\rangle$ does not correspond to a base time scale $s_{b}$ of the drive system. As to large time scales (corresponding to low modulation frequencies), their wavelet power spectra are essentially different. The wavelet spectrum of the response system in the area of the time scale $s_{m}$ is continuous and homogeneous, i.e. the modulation oscillations are characterized by continuous noise-like spectrum. For small values of coupling coefficient $R$, the shape of wavelet power spectrum changes [see Fig.4(b)). However, time scale synchronization is not observed, which is apparent from Fig. 3 (a).

With the increase of $R$ the conditions of TSS (6) and (77) are satisfied only by the time scales $s$ whose energy in power spectra of the drive and response systems is rather large. Really, at the values of coupling coefficient $R \approx$ 0.3 as it was already considered above, the conditions of TSS are fulfilled only for time scales near the basic scale $s_{b}=1 / f_{b} \approx 1.0$ (see also Fig. 2). As it follows from Fig.4(c), in the range of time scales marked in Fig.4(c) by grey color and symbol $S$, the energy of wavelet power spectrum is maximum.

From Fig.4(c) one can also see that the energy of wavelet spectrum, being fallen on the time scale $s_{m}$ of the response system simultaneously increases. However, at $R \approx 0.3$ its energy is not large enough, and TSS of field modulation oscillations is not observed.

At large coupling coefficients $R>0.35$ the shape of wavelet power spectra of output field oscillations of response and drive systems become close to each other (see Fig.4(d)). Base time scales $s_{m}$ and $s_{b}$ are presented in the power spectra with approximately equal intensity. The analysis of time scales dynamics shows, that the conditions of synchronization (6) and (17) are fulfilled for both most intensive time scales in wavelet power spectra and two ranges of time scales, in which phase locking occurs [see Fig.2 for $R>0.35$ ], are accordingly selected.

Thus, the TSS of two coupled chaotic spatially extended systems is observed first of all for those time scales $s$ whose energy in the chaotic power spectrum is great.

With the increase of coupling parameter the range of synchronized time scales $\left[s_{1}, s_{2}\right]$, for which the TSS conditions (6) and (7) are satisfied, is expanded. Time scales which are close to the most intensive scales in wavelet power spectrum and whose energy is large enough, start to be involved in synchronous dynamics.

The latter is well illustrated by Fig.2 from which it can be seen, that for both base time scales $s_{b}$ and $s_{m}$ extension of the range of phase locked scales $\Delta s$ with the increase of $R$ is observed. At large values of coupling coefficient $R$ the range of synchronous scales $\Delta s_{b}=s_{2 b}-s_{1 b}$ in the area of the base scale $s_{b}$ ceases to be expanded and, moreover, is narrowed down at $R>0.5$. At the same time in the area of scales $s_{m}$, describing modulation of amplitude of the output field of BWO, the range of synchronous scales $\Delta s_{m}=s_{2 m}-s_{1 m}$ increases linearly with the growth of $R$, at the expense of growth of 
top bound of area of synchronized scales $s_{2 m}$. At last we must note that with growth of $R$, the energy of the wavelet power spectrum of a response system is redistributed in such a manner that the increase of energy of larger scales $s>s_{m}$ is observed. Then the most intensive time scales in wavelet spectrum appear locally, near the top bound $s_{2 m}$ of synchronized time scales, therefore these time scales are first to be synchronized with the increase of coupling coefficient $R$.

\section{MEASURE OF SYNCHRONIZATION OF SPATIALLY EXTENDED ELECTRON-WAVE SYSTEM}

Introducing continuous set of time scales $s$ and the instantaneous phases associated with them, as well as separation of the range of synchronous time scales $\Delta s=$ $s_{2}-s_{1}$ allows us to inject the quantitative performance of a measure of chaotic synchronization of coupled systems [24, 27]. This measure $\gamma$ can be defined as a part of wavelet spectrum energy being fallen on the synchronized time scales

$$
\gamma=\frac{1}{E_{2}} \int_{s_{m}}^{s_{b}}\left\langle E_{2}(s)\right\rangle d s
$$

where $\left[s_{m} ; s_{b}\right]$ is the range of time scales for which the condition (6) is fulfilled and $E_{2}$ is the full energy of the wavelet spectrum

$$
E_{2}=\int_{0}^{+\infty}\left\langle E_{2}(s)\right\rangle d s
$$

of the response system.

The energy distribution over different time scales is a very useful characteristics for complex system behavior description. It is widely used in different applications (see e.g., [45, 46]).

This measure $\gamma$ is equal to zero for the nonsynchronized oscillations. $\gamma \neq 0$ means that in the coupled systems the conditions of TSS (6) and (7) are implemented. The value $\gamma=1$ shows, that oscillations in each of subsystems are identical or shifted upon some lag of time $T_{\tau}$. This is a complete or lag chaotic synchronization [4]. Increase of $\gamma$ from 0 up to 1 testifies to the increase of a part of the wavelet spectrum energy, being fallen on synchronous time scales $s$. Actually, the measure $\gamma$ characterizes how close to each other the chaotic oscillations are in both spatially extended systems.

In our case when the synchronous behaviour is observed for two base scales $s_{m}$ and $s_{b}$, it is possible to introduce measures of synchronization $\gamma_{b}$ and $\gamma_{m}$ according to each of time scales $s_{b}$ and $s_{m}$, and also the integral performance of the TSS measure of the systems: $\gamma=\gamma_{b}+\gamma_{m}$. Dependence of the measure $\gamma$ on the coupling coefficient

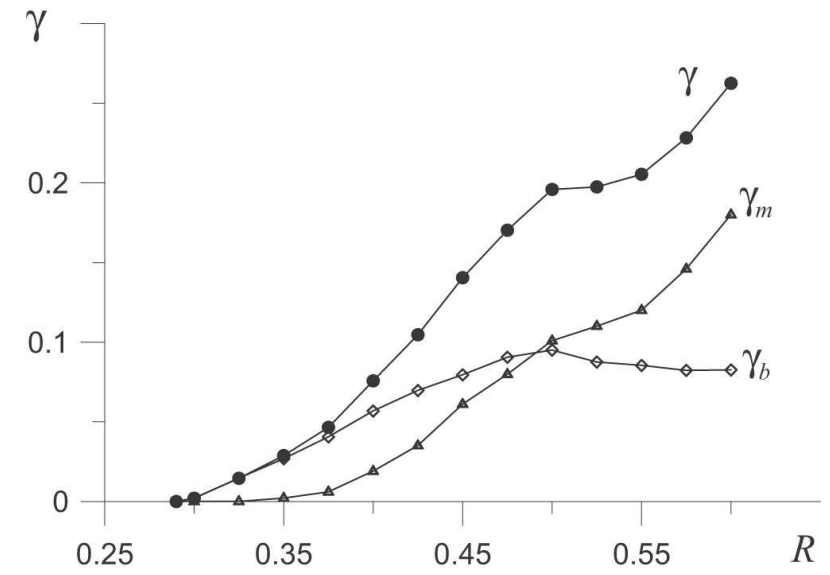

FIG. 5: The dependence of the synchronization measures $\gamma_{b}$, $\gamma_{m}$ and $\gamma$ of the response system on the coupling strength $R$

$R$ characterizes degree of chaotic synchronization of oscillations in the electron-wave systems with a backward wave.

In Fig. 5 the dependence of the synchronization measures $\gamma_{b}, \gamma_{m}$ and $\gamma$ for the response systems upon the coupling parameter $R$ is shown. It is obvious, that at small $R<0.4 \div 0.5$ the main part of energy of the wavelet power spectrum, being fallen at synchronized time scales, corresponds to the base scale $s_{b}\left(\gamma_{b}>\gamma_{m}\right)$.

Thus, in the coupled systems there is the capture of phases of time scales $s \sim s_{b}$ close to the time scale corresponding to the frequency of synchronism of electronic and electromagnetic waves. With further growth of $R$ the capture of frequencies close to the modulating frequency $f_{m}$ of the output signal of the driving BWO takes place. With the increase of $R$ the range of the synchronized scales describing low-frequency chaotic modulation of the output signal also increases. Hence, $\gamma_{m}$ (the normalized part of energy of synchronized scales corresponding to low-frequency modulation oscillations) grows with the increase of $R$. At $R>0.5$ part of energy of synchronous low-frequency modulation time scales $\gamma_{m}$ becomes more than the value $\gamma_{b}$, describing part of energy of synchronous high-frequency oscillations.

The integral part of energy $\gamma$, being fallen on synchronous time scales, monotonically increases with the growth of value of coupling coefficient $R$ [see Fig.5]. However, even at large values of $R$, when the response active medium is hardly influenced by the signal of the driving system $\left(R^{2} \sim 0.3 \div 0.4\right)$, the part of the energy, being fallen upon synchronous time scales, does not exceed value $\gamma \sim 0.3$.

Thus, at chaotic synchronization of two unidirectional coupled BWO at the increase of coupling coefficient, we can observe TSS near the most intensive time scale $s_{b}$ corresponding to frequency $f_{b}$ of synchronism of electronic and electromagnetic waves. With the growth of $R$ there is an increase of energy of synchronized scales in the area of the base scale $s_{b}$. At the same time, a range of synchro- 
nized time scales $\Delta s_{m}$, corresponding to low-frequency modulation of oscillations of field $F$, appears.

With the further increase of $R$ the growth of wavelet power spectrum energy, being fallen on synchronous time scales, is due only to the increase of the range of synchronized time scales $\Delta s_{m}$. The given behaviour of two unidirectional coupled systems is quantitatively described by the measure of chaotic synchronization $\gamma=\gamma(R)$.

\section{SPACE-TIME DYNAMICS OF RESPONSE CHAOTIC ELECTRON-WAVE SYSTEM}

Let us consider the physical processes in the nonautonomous (response) active medium when the chaotic synchronization appears. First of all, we are interested in space dynamics of response active medium.

The external chaotic signal generated by the drive distributed system influences on the point with coordinate $\xi=L$ of the response active medium and then spreads towards electron wave to the output $\xi=0$ border. The dimensionless length of the active media denoted as $L$ is equal to 1 . The synchronization of the whole active electron-wave system discussed above means the time scale synchronization of external signal $F_{\text {ext } 1}(\tau)$ and output signal $F_{\text {out } 2}(\tau)=F_{2}(\tau, \xi=0)$ of response media takes place. To consider the space-time dynamics of response system we have analyzed the presence of time scale synchronization between the external signal and the time series $F_{2}(\tau, \xi)$ obtained from different points $0<\xi<L$ of interaction space.

Let us consider the synchronization on time scales which are close to the most intensive scale $s_{b}$ of external chaotic signal wavelet spectrum. The synchronization of these time scales appears when the value of coupling strength $R$ is small enough and when the energy of external signal $P_{\text {ext }}=R^{2}\left|F_{\text {ext } 1}\right|^{2}$ is also small (see Section for detail). The range $\Delta s_{b}$ of synchronized time scales may be considered as a function of interaction space coordinate of active medium. This function $\Delta s_{b}=\Delta s_{b}(\xi)$ as well as the synchronization measure $\gamma=\gamma(\xi)$ (20) are the characteristics describing the space dynamics of chaotic synchronization appearance when the external chaotic signal acts on one of the border of the distributed system.

Fig. 6la) shows areas of synchronized time scales on the plane "coordinate $(L-\xi)$ - time scales $s$ " for three different values of coupling strength $R$. In Fig. [6) one can see that the width of the synchronized time scales area is maximum in the points of interaction space which are close to the border of system on which the chaotic signal of the drive active medium acts (see the border conditions (19) for the field $F_{2}$ of the response system). When the coordinate $\xi$ decreases, the area of synchronized time scales $\Delta s_{b}(\xi)$ converges gradually. The measure of synchronization $\gamma_{b}(\xi)$ behaves along the interaction space in the similar way [see Fig. [6(b)]. With the decreasing of $\xi^{-}$ coordinate the part of wavelet spectra energy being fallen on the synchronized time scales $\Delta s_{b}(\xi)$ also decreases.

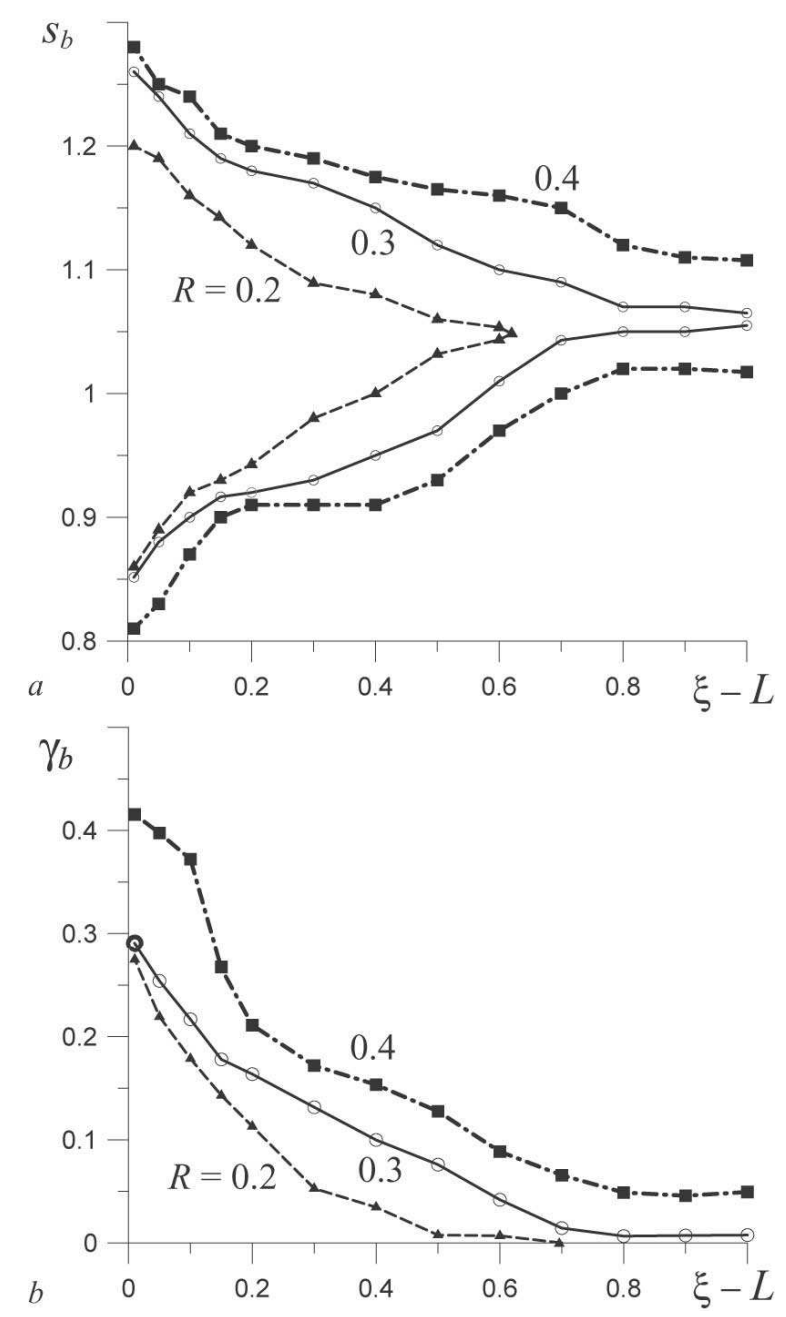

FIG. 6: (a) The areas of synchronized time scales on the plane "coordinate $(L-\xi)$ - time scales $s$ ". The coordinate $(L-\xi)$ is counted from the end of the response system. (b) The dependence of synchronization measure $\gamma_{b}$ on the coordinate of interaction space $(L-\xi)$. The black squares $(\mathbf{a})$ correspond to the value of coupling parameter $R=0.4$, circles (o) $R=0.3$ and black triangles $(\boldsymbol{\Delta})-R=0.2$

When the coupling strength $R$ is rather small $(R<$ 0.29 ) and the synchronization of two coupled active media is not observed [see Sections III] and IV], the width of the synchronized time scales area becomes equal to zero at the point of interaction space $\xi_{s}<L$. It means that the intricate space dynamics of phase locking takes place in the asynchronous regime. The dependence of the width of the synchronized time scales area on the coordinate $\xi$ (see also Fig. 6(a), case $R=0.2$ ) is described by

$$
\begin{array}{ll}
\Delta s \neq 0 & \xi>\xi_{s}, \\
\Delta s=0 & \xi<\xi_{s} .
\end{array}
$$

So, in this case it is possible to say that the interaction space of the response active medium is separated to two regions. 
First of them is characterized by length $L_{s}=L-\xi_{s}$ and abuts on the border $\xi=L$ of the response active system. The chaotic oscillations $F_{2}(\tau, \xi)\left(\xi \in\left(\xi_{s}, L\right)\right)$ in this region are synchronous (in terms of time scale synchronization) with the external chaotic signal $F_{\text {ext } 1}(\tau)$ generated by the drive system. Therefore, the synchronization measure is not equal to zero: $\left.\gamma_{b}\right|_{\xi>\xi_{s}} \neq 0$. This region of interaction space has naturally been called as "the region of synchronous oscillation". Its length $L_{s}$ has been called "length of synchronization".

In the second region $\xi \in\left(0, \xi_{s}\right)$ the chaotic synchronization is destroyed and the range of the synchronized time scales (22) as well as the synchronization measure is equal to zero.

With the increasing of $R$ the synchronization length also increases. For some value $R=R_{b}$ the length of synchronization becomes equal to the length of the interaction space $\mathrm{E}_{s} \equiv L$ (see Section IIII and Fig. 22). This means that in all space of response of electronwave medium the chaotic synchronization takes place and, therefore, one can say about synchronization of considered systems as whole. This regime of chaotic synchronization has been described above in sections [III and IV when the time scale dynamics of chaotic signal get from output $\xi=0$ of response system has been considered.

Fig. 6 (a) corresponding to the case of synchronization of the whole response active medium shows the borders of synchronized time scales $s$ along the interaction space. The value of coupling strength has been selected as $R=0.3 \approx R_{b}$. There is the range $\Delta s$ of synchronized time scales for every point $0 \leq \xi \leq L$. With coupling strength increasing the range of synchronized time scales also increases [see Fig. [6(a), $R=0.4$ ]. This result agrees well with Sec. III The value of wavelet power spectrum energy being fallen on synchronized time scales is equal approximately to $30 \div 40 \%$ in the point with coordinate $\xi=0.99 \mathrm{~L}$ and decreases to $1 \div 5 \%$ at the output border $\xi=0$ of the response active media.

In Fig. [7 the dependence of the length of synchronization $L_{s}$ on coupling parameter $R$ for the base time scale $s_{b}$ is shown (solid line). It is clear, that with the increasing of coupling strength the length of synchronization grows monotonically according to the linear law: $L_{s}(R) \sim R$. It follows, that with the growth of coupling coefficient $R$ we have the linear increase of the region where time scale synchronization near base scale $s_{b}$ is observed. Such behaviour of the response system is determined by the features of interaction of electronic and electromagnetic waves. Destruction of time scale synchronization is defined by violation of phase ratio between backward waves of electron current $I(\tau, \xi)$ and electromagnetic field $F(\tau, \xi)$.

So, at the fixed length of the response system, the phase shift $\Delta \varphi$ between field and current waves, originating due to the influence of external signal upon the response system, reduces on the length of synchronization $L_{s}$ to destruction of time scale synchronization on some time scale $s$. The phase shift is defined as $\Delta \varphi=$

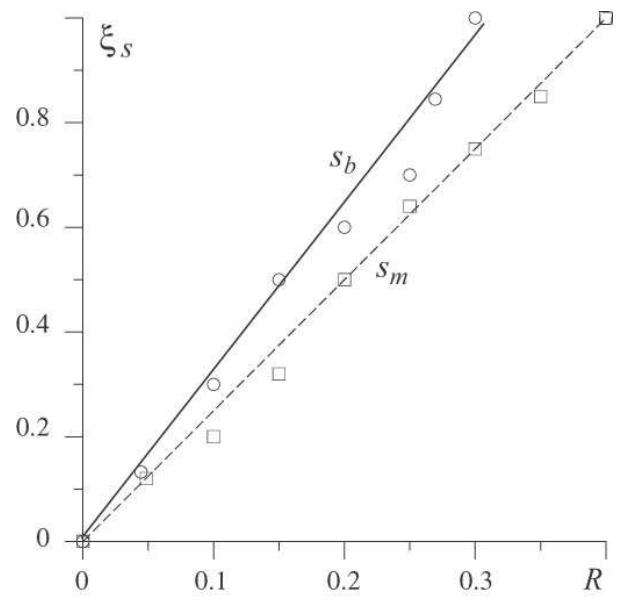

FIG. 7: Length of synchronization of response spatially extended system as function of coupling strength $R$. The dependence for base time scale $s_{b}$ is pointed by circles and approximation $L_{s} \sim R$ is plotted by solid line. The numerical dependence and approximation for modulating time scale $s_{m}$ is pointed by squares and dashed line

$2 \pi\left|f_{b}-f_{s}\right| \xi_{s} / v_{g}$, where $f_{b}=1 / s_{b}, f_{s}=1 / s$ and $v_{g}$ is the the group velocity of the backward electromagnetic wave.

On the other hand, dependence of the phase shift $\Delta \varphi$ upon the amplitude of the external field $R\left|F_{2}\right|$ can be presented as $\Delta \varphi=\kappa R F_{2}$, where $\kappa$ is the coefficient of proportionality depending on length of the response spatially extended system $A_{2}$. Comparing the estimated expressions for the phase shift $\Delta \varphi$ it is found out that the length of synchronization $L_{s}$ is proportional to $R$, as the numerical simulation shows (see Fig. 17).

It is important to note that curves shown in Fig. [6 correspond to the base time scale $s_{b}$ and time scales close to it. The similar results have been obtained for the space-time dynamics of scales which are close to the modulation time scale $s_{m}$ [see Fig. 7 dashed line]. The difference consists in that in this case the length of synchronized time scales area (length of synchronization) becomes equal to the system length for the bigger values of coupling strength than for the base time scale $s_{b}$. This also agrees well with the results discussed above.

\section{CONCLUSION}

In conclusion, in our article we have considered the chaotic oscillations in two unidirectionally coupled electron-wave active media. The approach of chaotic synchronization analysis proposed in our work [24, 27] has been used. This approach is based on the consideration of continuous set of time scales and chaotic signal phases associated with them. The application of this method allows us to investigate the chaotic synchronization of distributed active media demonstrating the intricate dynamics whereas the traditional methods of chaotic 
signal phase introduction fail due to the complex topology of the attractor. At the same time we succeed in dividing the chaotic synchronization into synchronization of low-frequency modulation oscillations of electromagnetic field and high-frequency generation one. One can see that it was impossible to do it using traditional approaches of chaotic synchronization analysis. So, the results described in our work allow to understand more clearly the common mechanisms of chaotic synchronization and justify the efficiency of new TSS-approach to chaotic synchronization analysis.

The main results are the following. With the increase of coupling strength the chaotic synchronization regime appears and is characterized by phase relationship between time scales of chaotic oscillations of drive and response active media. First of all, the synchronization of time scales corresponding to high-frequency spectral component appears. The synchronization of time scales corresponding to low-frequency oscillations takes place with the larger values of coupling strength $R$. When the chaotic synchronization of the whole active medium is not observed the interaction space may be divided into two regions corresponding to synchronous and asynchronous oscillations, respectively. The length of synchronous oscillation area $L_{s}$ grows when the coupling strength $R$ increases. The appearance of chaotic synchronization of two unidirectionally coupled active media is determined by condition of equality of synchronization length and length of the system.

It should also be noted that the method of investigation of chaotic synchronization of two coupled distributed systems discussed above can be easily applied to the analysis of higher spatial dimensions systems, such as 2D and $3 \mathrm{D}$ ones. In this case one have to analyze time series $x(t, \mathbf{r})$ obtained from different points $\mathbf{r}$ of space in the manner discussed above.

\section{ACKNOWLEDGMENTS}

We express our appreciation to Professor Dmirtiy I. Trubetskov for valuable discussions. We also thank Dr. S.V. Eremina for language support. This work has been supported by U.S. Civilian Research \& Development Foundation for the Independent States of the Former Soviet Union (CRDF, grant REC-006) and Russian Foundation for Basic Research (grant 05-02-16273). We also thank "Dynasty" Foundation.
[1] Rosenblum M.G., Pikovsky A.S., Kurths J., Phys. Rev. Lett. 76, 1804 (1996).

[2] Pikovsky A., Rosenblum M., Kurths J., Int. J. Bifurcation and Chaos 10, 2291 (2000).

[3] Boccaletti S., Kurths J., Osipov G., Valladares D.L., Zhou C., Physics Reports 366, 1 (2002).

[4] Pikovsky A., Rosenblum M., Kurths J., Synhronization: a universal concept in nonlinear sciences (Cambridge University Press, 2001).

[5] Ticos C.M., Rosa E., Pardo W.B., Walkenstein J.A., Monti M., Phys. Rev. Lett. 85, 2929 (2000).

[6] Anishchenko V.S., Balanov A.G., Janson N.B., Igosheva N.B., Bordyugov G.V., Int. J. Bifurcation and Chaos 10, 2339 (2000).

[7] Tass P.A. et al., Phys. Rev. Lett. 90, 088101 (2003).

[8] Prokhorov M. at al., Phys. Rev. E 68, 041913 (2003).

[9] Murali K., Lakshmanan M., Phys. Rev. E 48, R1624 (1994).

[10] Yang T., Wu C.W. and Chua L.O., IEEE Trans. Circuits and Syst. 44, 469 (1997).

[11] Liu Yaowen, Ge Guangming, Zhao Hong, Wang Yinghai, Phys. Rev. E 62, 7898 (2000).

[12] Garcia-Ojalvo J., Roy R., Phys.Rev.Lett. 86, 5204 (2001).

[13] Kurths J., Pikovsky A.S., Chaos Solit. Fract. 5, 1893 (1995).

[14] Boccaletti S., Bragard J., Arecchi F.T., Phys. Rev. E. 59, 6574 (1999).

[15] Bragard J., Arecchi F.T., Boccaletti S., Int. J. Bifurcation and Chaos 10, 2381 (2000).

[16] Ticos C.M., Rosa E., Pardo W.B., Walkenstein J.A., Monti M., Phys.Rev.Lett. 85 (2000).
[17] Ahlers V., Pikovsky A., Phys.Rev.Lett. 88, 254101 (2002).

[18] Valladares D.L., Boccaletti S., Feudel F., Kurths J., Phys. Rev. E 65, 055208 (2002).

[19] Mendoza C., Boccaletti S., Politi A., Phys. Rev. E. 69, 047202 (2004).

[20] Pecora L.M., Carroll T.L., Jonson G.A., Mar D.J., Chaos 7, 520 (1997).

[21] Boccaletti S., Valladares D.L., Phys. Rev. E 62, 7497 (2000).

[22] Anishchenko V.S., Vadivasova T.E., Journal of Communications Technology and Electronics 47, 117 (2002).

[23] Anishchenko V.S., Vadivasova T.E., Journal of Communications Technology and Electronics 49 (2004).

[24] Hramov A.E., Koronovskii A.A., Chaos 14, 603 (2004).

[25] Osipov G.V., Hu B., Zhou C., Ivanchenko M.V., Kurths J., Phys.Rev.Lett. 91, 024101 (2003).

[26] Romano M.C., Thiel M., Kurths J., von Bloh W., Phys. Lett. A 330, 214 (2004).

[27] Koronovskii A.A., Hramov A.E., JETP Lett. 79, 316 (2004).

[28] Daubechies I., Ten lectures on wavelets (SIAM, 1992).

[29] Kaiser G., A friendly guide to wavelets (Springer Verlag, 1994).

[30] Koronovskii A.A., Hramov A.E., Continuous wavelet analysis and its applications (In Russian) (Moscow, Fizmatlit, 2003).

[31] Grossman A. and Morlet J., SIAM J. Math. Anal. 15, 273 (1984).

[32] Soohoo R.F., Microwave Electronics (Addison-Wesley Longman, 1971).

[33] Infrared and Millimeter Waves (Academic Press, New 
York, 1983), K. Button ed.

[34] Granatstein V.L., Alexeff I., High Power Microwave Sources (Artech House Microwave Library, 1987).

[35] Felch K.L., Danly B.G., Jory H.R. et al, Proceedings IEEE 87, 752 (1999).

[36] Bezruchko B.P., Kuznetsov S.P., Trubetskov D.I., JETP Lett. 29, 162 (1979).

[37] Ginzburg N.S., Kuznetsov S.P., Fedoseeva T.N., Radiophys. Quantum Electron 21, 728 (1979).

[38] Levush B., Antonsen T.M., Bromborsky A., Lou W.R., Carmel Y., IEEE Trans. Plasma Sci. 20, 263 (1992).

[39] Nusinovich G.S., Vlasov A.N., Antonsen T.M., Phys.Rev.Lett. 87, 218301 (2001).

[40] Dronov V., Hendrey M.R., Antonsen T.M., Ott E., Chaos 14, 30 (2004).
[41] Trubetskov D.I., Mchedlova E.S., Anfinogentov V.G., Ponomorenko V.I., Ryskin N.M., Chaos 6, 358 (1996).

[42] Trubetskov A.E., Hramov A.E., Lectures on microwave electronics for physicists (In Russian) (Vol. 1. Fizmatlit, Moscow, 2003).

[43] Packard N.H., Crutchfield J.P., Farmer J.D., Shaw R.S., Phys. Rev. Lett. 45, 712 (1980).

[44] Takens F., in Lectures Notes in Mathematics, edited by Rand D., Young L.-S (N.Y.: Springler-Verlag, 1981), p. 366.

[45] Schwarz U., Kurths J., Kliem B., Krüger A., Urpo S., Astron. Astrophys., Suppl. Ser. 127 (1998).

[46] Wavelets in Physics (Cambridge University Press, 2004), J.C. Van den Berg ed. 